\title{
Can Hba1c be a marker for cardiovascular risk in type 2 Diabetes Mellitus
}

\author{
Deshmukh $S^{1}$, Singh $\mathrm{VB}^{2}$, Chetan Kumar $\mathrm{Hb}^{3}$, Meena $\mathrm{BL}^{4}$, Beniwal $\mathrm{S}^{5}$, Saini VK ${ }^{6}$ \\ ${ }^{1}$ Dr Sreehari Dshmukh, Post graduate student, Department of medicine, ${ }^{2}$ Dr V B Singh, Professor and Head of Geriatric \\ division, Department of medicine, ${ }^{3}$ Dr Chetan Kumar Hb, Post graduate student, Department of medicine, ${ }^{4}$ Dr Babulal \\ Meena, Assistant professor, Department of medicine, ${ }^{5}$ Dr Sanjay Beniwal, Assistant professor, Department of medicine, ${ }^{6}$ Dr \\ Vishnu Kumar Saini, Post graduate student, Department of medicine. All are affiliated with S P Medical College Bikaner, \\ Rajasthan, India
}

Address for Correspondence: Dr Sreehari Dshmukh, Email: sreeharideshmukh@gmail.com

Introduction: One in every five Indians in geriatric age has diabetes. Diabetes is associated with increase in TG and apo B, with decrease in HDL component, so it contributes to atherosclerosis formation. We conducted a study to correlate glycaemic control using glycated haemoglobin with dyslipidaemia. Methods: The study is a cross sectional study with 200 diabetic patients, $\mathrm{HbA}_{1 \mathrm{c}}$ was correlated with lipid profile and atherogenic index of plasma (AIP). AIP is log ratio of plasma triglyceride to HDL. Patients were categorised into good glycaemic control $(\leq 7 \%)$ and poor glycaemic control $(>7 \%)$ based on glycaemic control with $\mathrm{HbA}_{1} \mathrm{C}$ as the marker. Results: Study showed the duration of diabetes directly correlates with HbA1c. None of the patients who had diabetes for more than 10 had $\mathrm{HbA}_{1 \mathrm{c}}$ less than seven. BMI had direct association with HbA1c. $\mathrm{HbA}_{1} \mathrm{c}$ demonstrated a positive significant correlation with Total Cholesterol, LDL and a negative significant correlation with HDL. Atherogenic index of plasma directly correlates with $\mathrm{HbA}_{1 \mathrm{c}}$ with mean AIP of $0.36+0.24$ and $0.58+18$ in good glycaemic control (GCC) and poor glycaemic control (PCC) respectively. Patients with $\mathrm{HbA} 1 \mathrm{c}>7.0 \%$ had statistically significantly higher value of total cholesterol, LDL when compared with $<7.0 \%$. Conclusion: These findings clearly indicate that $\mathrm{HbA}_{1} \mathrm{c}$ can provide valuable supplementary information about the extent of dyslipidaemia, AIP. Screening for $\mathrm{HbA}_{1} \mathrm{c}$ estimation helps in preventing complications by achieving adequate glycaemic control. Thus, $\mathrm{HbA}_{1 \mathrm{c}}$ can be used as a potential biomarker to identify patients with cardiovascular risk in Type 2 Diabetes Mellitus and can used as a guide for aggressive therapeutic approach.

Key words: Diabetes, Atherogenic index, Dyslipidemia.

\section{Introduction}

Type 2 diabetes is associated with insulin resistance in the target organ with hyperinsulinemia initially and loss of beta islet cell in later stages which leads to insulin deficiency. Incidence of diabetes now has exceeded what was expected 10 years ago and it's expected to increase further. Prevention of diabetes is of utmost importance for the primary care physicians. Patient can be asymptomatic for a long time before the diagnosis is made [1]. Diabetes is associated with many vascular complications. Alterations in lipid and lipoprotein profile contribute to atherosclerosis in type 2 diabetes. The atherogenic index of plasma (AIP), defined as logarithm [log] of the ratio of plasma concentration of triglycerides to high-density lipoprotein (HDL) cholesterol, has recently been proposed as a predictive marker for plasma atherogenicity and is positively correlated with cardiovascular disease risk [2].

Manuscript received: $13^{\text {th }}$ Apr 2015

Reviewed: $22^{\text {th }}$ Apr 2015

Author Corrected: $26^{\text {th }}$ Apr 2015

Accepted for Publication: $25^{\text {th }}$ May 2015
The aim of the study is to study the correlation of glycaemic control using glycated haemoglobin (HbA1c) with diabetic dyslipidaemia and atherogenic index of plasma. This investigation is an attempt to evaluate the diagnostic value of $\mathrm{HbA1c}$ in predicting cardiovascular risk by studying its correlation with atherogenic dylipidemia, Atherogenic Index of Plasma with $\mathrm{HbA}_{1} \mathrm{C}$

\section{Methods and Materials}

Present study was conducted in S.P. Medical College \& Associated Group of P.B.M. Hospitals, Bikaner. It was a cross sectional study and enrolled 200 Type 2 Diabetes patients attending medical outdoor, Geriatric care and research centre and those admitted in hospital. Before enrolment, details about nature and utility of present study were explained to all patients and informed consent was taken. All patients were subjected to detailed clinical examination and relevant investigations. Only after the 


\section{Case Report}

inclusion and exclusion criteria were met, the subjects were included in the study.

Patients were classified into two groups depending on their glycated hemoglobin (HbA1c); Good Glycemic Control (GGC) group having $\mathrm{HbA} 1 \mathrm{c}<7.0 \%$ and Poor Glycemic Control (PGC) group having HbA1c $>7.0 \%$. For serum lipid reference level, National Cholesterol Education Programme (NCEP) Adult Treatment Panel III (ATP III) guidelines were referred [3]. According to NCEP-ATP III guideline, hypercholesterolemia is defined as Total Cholesterol $(\mathrm{TCH})>200 \mathrm{mg} / \mathrm{dl}$, high LDL when value > $100 \mathrm{mg} / \mathrm{dl}$, hypertriglyceridemia as $\mathrm{TG}>150$ $\mathrm{mg} / \mathrm{dl}$ and low HDL when value $<40 \mathrm{mg} / \mathrm{dl}$ in men and < $50 \mathrm{mg} / \mathrm{dl}$ in women. Dyslipidemia will be defined by presence of one or more than one abnormal serum lipid concentration. The atherogenic index of plasma (AIP), which is a predictive marker for plasma atherogenicity were measured as logarithm [log] of the ratio of plasma concentration of triglycerides to high-density lipoprotein (HDL) cholesterol with AIP $<0.11$ low risk; AIP 0.11 0.21 intermediate risk and AIP $>0.21$ increased risk. Venous blood samples were collected from all the subjects after at least 8 hours of fasting.

Diabetics with Family history of hyperlipidemia, with heart failure, respiratory, neurological, renal and malignant disorders, on lipid lowering therapy, thiazolidinediones and anti-inflammatory drugs, with abnormal liver function tests, with acute febrile illness, asymptomatic infections and chronic illnesses were excluded from the study. Statistical analysis was performed using the Statistical Package for Social Sciences (SPSS, version 17). The $\mathrm{P}$ values $<0.05$ were considered statistically significant.

\section{Results}

Two hundred Type 2 Diabetes Mellitus patients comprising all age groups were recruited from the outpatient and inpatient department. There were 117 patients with glycated hemoglobin $>7$ and 83 patients with glycated hemoglobin <7. Following observations were made. Seventy patients had elevated total cholesterol levels with 54 patients $(77.1 \%)$ with $\mathrm{HbA1c}>7$, resulting in significant correlation between $\mathrm{Hbalc}$ and total cholesterol levels. Eighty one type $2 \mathrm{DM}$ patients had elevated TG levels $(>150 \mathrm{mg} / \mathrm{dl})$ with 69 patients $(85.1 \%)$ with $\mathrm{HbA} 1 \mathrm{c}>7$, resulting in significant correlation between Hba1c and serum triglyceride levels. About 132 type $2 \mathrm{DM}$ patients had elevated LDL levels (>100mg/dl) with 79 patients $(59.8 \%)$ with $\mathrm{HbAlc}>7$, resulting in significant correlation between Hba1c and serum LDL levels. About 50 female type $2 \mathrm{DM}$ patients had decreased HDL levels with 24 patients $(48 \%)$ with $\mathrm{HbA} 1 \mathrm{c}<7$, resulting in no significant correlation between Hbalc and serum HDL levels in females. About 48 male type 2 DM patients had decreased HDL levels with 32 patients $(66.6 \%)$ with $\mathrm{HbA} 1 \mathrm{c}<7$ resulting in no significant correlation between Hbalc and serum HDL levels. Patients were divided into three groups based on Atherogenic Index Plasma (AIP) into Low, Intermediate and Severe grades including 17, 18 and 165 patients respectively with 113 patients $(68.4 \%)$ with Hbalc levels $>7$ with the difference being highly significant $(\mathrm{p}<0.001)$. FBS showed that the mean FBS level in HbA1c $<7$ group was $130.63+32.95$ and the mean FBS in HBA1c $>7$ group is $174.78+66.18$, with the FBS levels showing a significant correlation with the glycated hemoglobin. Distribution of the cases according to the age group showed 94 patients in 6-10 yrs group with 51 patients $(54.2 \%)$ in HbA1c $>7$. The age groups $11-15$ and $>15$ had all patients $(100 \%)$ in $\mathrm{HbA} 1 \mathrm{c}>7$ group resulting in significant correlation of duration of diabetes with glycated hemoglobin. Out of total 83 patients with HbA1c $<7 \mathrm{gm} \%, 42$ patients $(50.60 \%)$ had normal BMI. In HbAlc $>7$ group, 3, 75, 16 and 23 patients were from underweight, normal, overweight and obese group and the difference was statistically significant $(\mathrm{p}<0.05)$. Out of total 69 females, 50 females had their WHR $>0.85$ with 32 females $(64 \%)$ with HbA1c $>7$. In males, 28 had their WHR $>0.95$ with $24(85.71 \%)$ of them $\mathrm{HbA} 1 \mathrm{c}<7$. On statistical analysis, the difference was statistically highly significant $(\mathrm{p}<0.001)$. Distribution of cases according to residence showed that majority of patients belonged to rural areas with a total of 133 patients in which about 86 patients $(64.66 \%)$ with $\mathrm{HbAlc}>7$. In the HbA1c $<7$ group, 36 of the total 67 patients $(53.73 \%)$ belonged to urban areas with significant correlation of glycemic control with patients of rural residence. Distribution of cases according to the socioeconomic status show that 157 patients (78.5\%) belong to the lower and middle SES group out of which 82 patients $(52.22 \%)$ belong to the poor glycemic control group with glycated hemoglobin showing significant positive correlation with socioeconomic status which may be due to the inability of the patients with lower incomes to afford adequate healthcare facilities which leads to poor glycemic control. 
Table 1: Association of various parameters with Glycaemic control

\begin{tabular}{|l|l|l|l|}
\hline Parameter & Good glycaemic control & Poor glycaemic control & P value \\
\hline Duration of diabetes & $5.54 \pm 2.87$ & $8.91 \pm 5.22$ & $<0.001$ \\
\hline FBS & $130.6 \pm 32.9$ & $174.7 \pm 66.1$ & $<0.001$ \\
\hline BMI & $22.8 \pm 2.09$ & $27.01 \pm 5.06$ & $<0.001$ \\
\hline TC & $173 \pm 32.5$ & $190.6 \pm 34.9$ & $<0.001$ \\
\hline TG & $116.6 \pm 95.9$ & $166.2 \pm 54$ & $<0.001$ \\
\hline HDL-C & $43.6 \pm 6$ & $42.08 \pm 7.51$ & 0.11 \\
\hline LDL-C & $106.3 \pm 26.3$ & $115.2 \pm 31$ & $<0.05$ \\
\hline AIP & $0.36 \pm 0.24$ & $0.58 \pm 0.18$ & $<0.001$ \\
\hline
\end{tabular}

\section{Discussion}

The prevalence of diabetes is increasing rapidly all over the world in last 20 years. India is often called the diabetic capital of the world as it about to overtake China in terms of prevalence. According to reports, in 2011 there were 366 million people with diabetes all over the world and it is expected to rise to 552 million by 2030 which is almost $80 \%$ increase [4]. The morbidity of diabetes is due to its complications which involves both macrovascular (stroke, peripheral vascular disease and coronary artery disease) as well as microvascular components (nephropathy, neuropathy and retinopathy) [5].

The percentage of glycosylated haemoglobin (HbA1c) reflects the glycaemic control of a patient during the 8-10 week period. The Diabetes complications and control trial (DCCT) established HbA1c as the gold standard of glycaemic control. Lowering $\mathrm{HbA} 1 \mathrm{C}$ is one of the aims in diabetic treatment as it reduces microvascular and it also helps in controlling macrovascular complications if implemented early in the disease [6].

Major risk factor for the development of cardiovascular events in diabetes is dyslipidaemia. The classic features of diabetic dyslipidaemia are high plasma triglyceride concentration, low HDL cholesterol component and increased concentration of small dense LDL-cholesterol particles [7]. The LDL can be normal in diabetes. The LDL cholesterol found in diabetes is small dense molecule which is more atherogenic. Diabetics display enhanced LDL oxidizability and there is increased rate of atherosclerosis [8,9]. There is local release of hypochlorous acid from myeloperoxidase, which interacts with HDL molecule and decreases its action which is reverse cholesterol transport. There are various alterations in small dense LDL molecule which make it more atherogenic like reduced LDL receptor affinity[10], greater propensity for transport into the subendothelial space[11], increased binding to arterial wall proteoglycans. So it is prudent to prescribe statins to elderly diabetics even if the LDL component is within normal limits. Hyperglycemia causes increased activity of hepatic lipase that leads to increased clearance of HDL while impaired catabolism of VLDL causes decreased formation of HDL, which is one of the reasons why HDL$\mathrm{C}$ levels are low in type 2 diabetes [12]. The other reasons for lowered HDL are probably most diabetics are obese and may be due to high triglycerides. HDL cholesterol is inversely correlated with cardiovascular risk [13]. Mild hyperglycemia leads to increased LDL production while insulin resistance or relative insulin deficiency causes defects in LDL clearance; thus the LDL cholesterol levels increase. Both dyslipidemia and $\mathrm{HbA}_{1} \mathrm{C}$ are independent risk factors for developing cardiovascular events. Lowering $\mathrm{HbA}_{1} \mathrm{C}$ will certainly lower the risk of having CVD later in life. It has been shown that reducing the HbA1c level by $0.2 \%$ could lower the mortality by $10 \%$ [14]. Is there an effect of age and duration of diabetes on lipid profile is an topic for discussion, nothing has been proved as of now. Some studies report there is clear association while few other studies negate this idea $[15,16]$.

The Atherogenic index of plasma (AIP) has recently been proposed as a marker of plasma atherogenicity and is positively correlated with cardiovascular disease risk[2]. In view of the predisposition for the development of atherosclerotic vascular disease in the diabetics, clinicians give importance to good glycaemic and cholesterol control in the patients. Triglycerides and HDL-cholesterol in AIP reflect the balance between the atherogenic (bad cholesterol) and protective (good cholesterol) lipoproteins. AIP correlates with the size of pro- and antiatherogenic lipoprotein particles. Clinical studies have shown that AIP predicts cardiovascular risk. AIP reflects the delicate metabolic interactions within the whole lipoprotein complex[17].

So what is the target HbA1c? Lowering A1C to below or around $7 \%$ as already been said it reduces both microvascular as well as macrovascular complications of diabetes. So, clinician should be advising patients to keep 


\section{Case Report}

their HbA1c levels below 7\% (in a non-pregnant adults). In gestational pregnancy the the $\mathrm{HbA} 1 \mathrm{c}$ target is around $6 \%$. Less stringent HbA1c goals $(<8 \%)$ in patients with a history of severe hypoglycemia, has limited life expectancy, advanced microvascular or macrovascular complications, hypoglycaemia unawareness and extensive comorbid conditions and in those with long-standing diabetes in whom the general goal is difficult to attain despite diabetes self-management education (DSME), and effective doses of multiple glucose-lowering agents including insulin[18].

\section{Conclusion}

These findings clearly indicate that $\mathrm{HbAlc}$ can provide valuable supplementary information about the extent of atherogenic dyslipidemia, Atherogenic Index of Plasma, besides its primary role in monitoring long-term glycemic control. Therefore, regular screening for $\mathrm{HbAlc}$ estimation can help in clinical management to prevent complications by achieving adequate glycemic control. Thus, HbA1c can be used as a potential biomarker to identify patients with cardiovascular risk and patients with incipient nephropathy in Type 2 Diabetes Mellitus.

Funding: Nil, Conflict of interest: None initiated.

Permission from IRB: Yes

\section{References}

1. American Diabetes Association. Diagnosis and Classification of Diabetes Mellitus. Diabetes Care January 2010 vol. 33 no. Supplement 1 S62-S69.

2. Chromium picolinate and biotin combination reduces atherogenic index of plasma in patients with type 2 diabetes mellitus: a placebo-controlled, double-blinded, randomizedclinical trial. Geohas $\mathbf{J}$ et al. Am J Med Sci. 2007 Mar;333(3):145-153.

3. Executive Summary of the Third Report of the National Cholesterol Education Program (NCEP) Expert Panel on Detection, Evaluation, and Treatment of High Blood Cholesterol in Adults (Adult Treatment Panel III). JAMA, May 16, 2001.Vol 285, No. 19:2486-2497.

4. Whiting DR et al. IDF diabetes atlas: global estimates of the prevalence of diabetes for 2011 and 2030. Diabetes Res Clin Pract. 2011 Dec;94(3):311-321.

5. Ronald K. Hyperglycemia and Microvascular and Macrovascular Disease in Diabetes, Diabetes Care, 1995; 18(2): 258-268.
6. Irene M S, Amanda I A, Andrew W N, David R M, Susan EM et al, Association of glycaemia with macrovascular and microvascular complications of type 2 diabetes (UKPDS 35): prospective observational study, BMJ , 2000;321:405-412.

7. Chintamani Bodhe, Deepali Jankar, Tara Bhutada et al. HbA1c: predictor of dyslipidemia and atherogenicity in diabetes mellitus. International Journal of Basic Medical Science - November 2011;2(5): 278-282.

8. Fonseca VA. Management of diabetes mellitus and insulin resistance in patients with cardiovascular disease. Am J Cardiol. 2003 Aug 18;92(4A):50J-60J.

9. Schwenke DC, D’Agostino RB Jr, Goff DC Jr, Karter AJ, Rewers MJ, Wagenknecht LE. Differences in LDL oxidizability by glycemic status: the insulin resistance atherosclerosis study. Diabetes Care. 2003 May;26(5):1449-55.

10. Campos H, Arnold KS, Balestra ME, Innerarity TL, Krauss RM: Differences in receptor binding of LDL subfractions. Arterioscler Thromb Vasc Biol. 1996 Jun;16(6):794-801.

11. Bjornheden T, Babyi A, Bondjers G, Wiklund O. Accumulation of lipoprotein fractions and subfractions in the arterial wall, determined in an in vitro perfusion system. Atherosclerosis. 1996 Jun;123(1-2):43-56.

12. Joslin's Diabetes Mellitus 14th Ed. Lippincott Williams \& Wilkins Chapter 33: Pathophysiology and treatment of lipid disorders in diabetes, pp. 567-571.

13. Ahmad Khan H. Clinical significance of HbA1c as a marker of circulating lipids in male and female type 2 diabetic patients. Acta Diabetol. 2007 Dec;44(4):193-200. Epub 2007 Sep 1.

14. Ram Vinod Mahato. Association between glycaemic control and serum lipid profile in type 2 diabetic patients: Glycated haemoglobin as a dual biomarker. Biomedical Research, 2011, pp. 375-380.

15. H.O. Otamere, C.P Aloamaka, PO.Okokhere , W.A. Adisa. Lipid Profile in Diabetes Mellitus; What Impact Has Age and Duration. Br. J. Pharmacol. Toxicol. 2011; 2(3): 135-137,

16. Amy SS, Lawrence M D, Thomas R K, Zhiqian G, Philip R K, Stephen R D, Elaine MU, Influence of Duration of Diabetes, Glycemic Control, and Traditional Cardiovascular Risk Factors on Early Atherosclerotic 
Vascular Changes in Adolescents and Young Adults with Type 2 Diabetes Mellitus. J Clin Endocrinol Metab. 2009; 94: 3740-3745.

17. Dobiasova M, Frohlich J, The plasma parameter log (TG/HDL-C) as an atherogenic index: correlation with lipoprotein particle size and esterification rate in apoB- lipoprotein-depleted plasma (FER(HDL), Clin Biochem, 2001; 34(7): 583-588.

18. Executive Summary of the 2014 American Diabetes Association Clinical Practice Recommendations (Diabetes Care 2014;37, suppl.1: S5-13.

\section{How to cite this article?}

Deshmukh S, Singh VB, Chetan Kumar Hb, Meena BL, Beniwal S, Saini VK. Can Hba1c be a marker for cardiovascular risk in type 2 Diabetes Mellitus. Int J Med Res Rev 2015;3(4):419-423. doi: 10.17511/ijmrr.2015.i4.083. 